\title{
Plan integral de fortalecimiento académico para mejorar la calidad del servicio Educativo
}

\author{
Integral plan for academic strengthening to improve the quality of the \\ Educational service
}

DOI: https://doi.org/10.33262/rmc.v6i2.1252

\author{
Glenda Cecibel Intriago Alcívar ${ }^{1}$ \\ Universidad Técnica de Babahoyo, Ecuador \\ (iD https://orcid.org/0000-0002-5360-9544 \\ glendaint@hotmail.com \\ Augusto Franklin Mendiburu Rojas ${ }^{2}$ \\ Universidad Técnica de Babahoyo, Ecuador \\ (iD https://orcid.org/0000-0002-2650-216X \\ amendiburur@utb.edu.ec \\ Marcos David Oviedo Rodríguez ${ }^{3}$ \\ Universidad Técnica de Babahoyo, Ecuador \\ https://orcid.org/0000-0001-8919-6123 \\ moviedo@utb.edu.ec
}

\section{RESUMEN}

La educación es sinónimo de desarrollo, por esto, el objetivo de la investigación es proponer un plan integral de fortalecimiento académico (PIFA) para mejorar la calidad del servicio educativo (CSE). La metodología utilizada se fundamenta en el tipo de investigación aplicada, con enfoque mixto, alcance descriptivo y explicativo, diseño no experimental. La muestra fue de 249 personas, a los que se aplicó un cuestionario previamente validado por expertos. Los resultados se situaron por encima del $50 \%$, respecto a la pertinencia de los procesos de planificación, ejecución y evaluación del PIFA. Los resultados obtenidos en la Prueba Chi Cuadrado, Normalidad y de Correlación, 
mostraron el valor de sig. $0.000<0.05$, con lo que se rechazó la hipótesis nula y se aceptó la hipótesis alternativa, con lo que, se concluyó que la elaboración, ejecución y evaluación del PIFA viabilizará la mejora de la CSE, centrándose en que el personal docente deberá contar con formación académica, experiencia profesional y capacitaciones pertinentes; los procesos de investigación formativa, producción científica y vinculación social, deberán potenciarse. En el diseño del PIFA se contempló la planificación, ejecución de estrategias educativas y evaluación de procesos, enmarcadas en modelos de gestión, que promoverán el mejoramiento continuo, para garantizar la CSE.

PALABRAS CLAVES: planificación, ejecución, evaluación, calidad, servicio educativo

\section{ABSTRACT}

Education is synonymous with development; therefore, the objective of the research is to propose a comprehensive plan for academic strengthening (PIFA) to improve the quality of the educational service (CSE). The methodology is based on the type of applied research, with a mixed approach, descriptive and explanatory scope, non-experimental design. The sample consisted of 249 people, to whom a questionnaire previously validated by experts was applied. The results were above $50 \%$, regarding the relevance of the planning, execution, and evaluation processes of the PIFA. The results obtained in the Chi Square Test, Normality and Correlation, showed the value of sig. $0.000<0.05$, with which the null hypothesis was rejected and the alternative hypothesis was accepted, then it was concluded that the preparation, execution and evaluation of the PIFA will make the improvement of the CSE feasible, focusing on the fact that the teaching staff must have academic training, relevant professional experience and training; the processes of formative research, scientific production and social bonding must be strengthened. In the design of the PIFA, planning, execution of educational strategies and evaluation of processes were contemplated, framed in management models, which will promote continuous improvement, to guarantee CSE.

KEYWORDS: planning, execution, evaluation, quality, educational service 


\section{INTRODUCCIÓN}

La educación es un derecho para todos los seres humanos, con ésta los sujetos obtienen conocimientos para desempeñar un rol fundamental en la sociedad, por lo cual la calidad del servicio educativo no se orienta solo a cumplir las actividades curriculares, sino también en la búsqueda de la excelencia. De acuerdo con Galván (2019) indica que uno de los problemas que está pasando México, es la motivación por la creación de 100 nuevas universidades gratuitas, decisión que ha sido cuestionada porque no se puede garantizar la calidad de este servicio, si no se cuenta con las condiciones y los recursos apropiados (párr. 1-4). Asimismo, el artículo denominado "El éxito del modelo educativo en Canadá" indica que, en la provincia de Ontario, la contratación de los profesores es garantía de calidad del servicio educativo (Torres, 2018, párr. 1-3). También, Becerra (2019) en su investigación en España manifiesta que los estudiantes deben desarrollar prácticas en empresas, que luego le permitan una incorporación sencilla al mundo laboral. Por otro lado, en España la inversión per cápita en investigación es baja o media-baja, esto limita que se desarrollen Grupos de proyectos de Investigación I+D+I, impidiendo la generación de investigaciones científicas a través de programas específicos o Semilleros de Investigación (párr.13-14).

En Ecuador, Montero (2018) menciona que se crearon normativas que reducen la autonomía universitaria, imponiendo el incremento del número de doctores con amplia experiencia en investigación y producción científica (párr. 2), asimismo, se han elaborado y puesto en práctica varios modelos de evaluación que orientan el cumplimiento de los objetivos institucionales, a través de los cuales se pretende mejorar aspectos como la generación y publicación de investigaciones por parte de los docentes universitarios (Consejo de Evaluación, Acreditación, y Aseguramiento de la Calidad de la Educación Superior, 2017, pág. 8). En el Informe de Autoevaluación del 6 mayo 2015 de la Universidad Técnica de Babahoyo, se identificaron las debilidades más significativas de los procesos sustantivos (Universidad Técnica de Babahoyo, 2016, pág. 4). Ante esta situación, sobre la condición de la Universidad de estudio, y la problemática identificada en cada elemento relacionado con el servicio educativo ofertado, es claro que no se está logrando la calidad, pues evidencia deficiencias de acuerdo con el informe arriba mencionado, esto nos lleva a formularnos la siguiente pregunta de investigación: ¿Cómo 

Ecuador 2020?

Al ser la calidad del servicio educativo una preocupación latente, por parte del estado, y tomando como referencia modelos de gestión ya comprobados, esta investigación se justifica en lo teórico porque contribuirá a fortalecer y afianzar el conocimiento como una estrategia de desarrollo, sustentado en el modelo de gestión de calidad total (TQM), y el modelo de excelencia (EFQM). Como aporte práctico, estos dos modelos son funcionales porque incidirán en la mejora de la calidad del servicio educativo. En el ámbito social, estos modelos cuentan con criterios y fundamentos que se han establecido como lineamientos generales para proyectarse a un sistema de calidad que tiene su impacto en cada área vinculada al desarrollo del proceso educativo universitario, como son docencia, investigación y extensión. Por tanto, se ha planteado como propósito de investigación proponer un plan integral de fortalecimiento académico para mejorar la calidad del servicio educativo en la Universidad Técnica de Babahoyo, y para el logro del propósito mencionado se plantean los siguientes objetivos específicos: 1) Diagnosticar el estado actual de la calidad del servicio educativo, 2) Identificar los factores influyentes en la calidad del servicio educativo, 3) Diseñar plan integral de fortalecimiento académico para mejorar la calidad del servicio educativo y 4) Analizar a través del juicio de expertos la pertinencia de la implementación del plan integral de fortalecimiento académico en la calidad del servicio educativo; así mismo, se plantea la siguiente hipótesis: la implementación de un plan integral de fortalecimiento académico mejoraría la calidad del servicio educativo en la Universidad Técnica de Babahoyo, Ecuador 2020.

\section{DESARROLLO}

Esta investigación se desarrolla considerando los procedimientos de la investigación científica, y habiendo realizado la revisión detallada de información referidos al servicio universitario desde una perspectiva de calidad. Es así, como en la investigación de Zhang et al. (2020), se destaca el rol de la educación como pilar de desarrollo, sin embargo, los gestores administrativos se ven limitados en su accionar debido a los escasos recursos económicos, por ello se presenta una propuesta de gestión que promueve la evaluación y prueba de toma de decisiones (DEMATEL), con lo que se determinan los indicadores clave de desempeño universitario (pág. 15). También Tyurikov et al. (2019) presentan un 
modelo de evaluación de la calidad y la demanda de servicios educativos, en el que se analiza la condición profesional de los graduados, y la situación de los programas educativos que buscan generar competencias profesionales (pág. 160). Ivanova et al. (2020) en el estudio del tema "Los objetivos y aspectos prácticos del sistema de aseguramiento de la calidad de la educación superior" afirman que la mayoría de los países buscan garantizar la calidad de la educación superior considerando los acelerados procesos globalizados, regulando las normativas, basados en las vivencias universitarias y casos prácticos que evidencian las debilidades (pág. 119). También Araujo (2016) sobre la pertinencia educativa, refiere que es un reto y un desafío que tiene actualmente el sistema educativo, en este contexto, indica que no se dejará de lado las políticas nacionales e internacionales, el contexto laboral que expone sus requerimientos, los actores del sistema de educación de otros niveles, el contexto cultural, y la opinión de estudiantes y docentes (párr. 12-14). Así mismo, Bournissen (2019) propone un modelo para interrelacionar los procesos administrativos, pedagógicos y tecnológicos que permitan modernizar el servicio educativo, concluyendo que "el e-learning incidirá en la posición de la universidad frente al avance tecnológico y a la sociedad misma" (pág. 205). Respecto a la calidad educativa en México, se evidencio la preocupación por parte de la comunidad universitaria sobre el entorno de aprendizaje, principalmente en el ámbito tecnológico, pues las instituciones de educación de educación superior no actualizan sus equipos, softwares y el acceso limitado al servicio de internet impide que docentes y estudiantes accedan a información actualizada que les permita concretar un proceso de enseñanza aprendizaje de calidad (Torres et al. 2017, pág. 19).

En otro aspecto Miyahira (2019), indica que para que se genere investigación científica, lo primero es incorporar asignaturas para el desarrollo de la investigación formativa (pág. 119). Del mismo modo, Rubio et al. (2015) en el estudio desarrollado en España, concluyen que la investigación formativa es un proceso educativo dinámico, que orienta al estudiante sobre la aplicación de métodos educativos, como él (APP) Aprendizaje Orientado a Proyecto (págs. 181-182). Espinoza (2016) también analiza los indicadores de gestión relacionados a la investigación como son: planta docente, investigación, gestión administrativa, normativas para estudiantes y entornos de aprendizaje que busca promover la mejora sistemática de la educación (pág.210), en esta misma línea, Barreno 
et al. (2018) en la investigación realizada en la Universidad Estatal de Milagro - Ecuador, sobre la vinculación con la sociedad, indican que no se aporta con alternativas de solución confiables a los problemas de la comunidad, sin embargo, los procesos de vinculación a través de las prácticas preprofesionales promueven el perfeccionamiento de los estudiantes que se convertirán en profesionales, concluyendo que las universidades se presentan a la comunidad interactuando e interviniendo estratégicamente a través de los estudiantes, promoviendo el desarrollo sociopolítico y cultural del contexto (págs. 40,41 y 44)

Por su parte, Romillo y Polaino (2019) aportan con una aplicación del enfoque de gestión tipo pirámide, desarrollado jerárquicamente en todos los procesos sustanciales de su dinámica como son: docencia, desarrollo investigativo y extensión universitaria con la comunidad, articulado logísticamente con el apoyo administrativo para obtener mejores resultados operativos, esto llevo a la Universidad de Otavalo a la condición de acreditada, por lo que los autores indican que: "esto deja abierta la posibilidad de analizar los procedimientos que se plantean en este modelo de gestión (pág. 11). Otro aspecto en el sistema educativo superior son los procesos pedagógicos, sobre estos, Castañeda (2015) indica que la selección de los docentes no es la apropiada, manifiesta que el maestro deberá evidenciar amplio conocimiento y dominio en el área pedagógica, para la articulación del macro, meso y micro currículo, que responde a un contexto social, y al no valorarse así, se estaría educando aisladamente a un objetivo educativo nacional (pág. 80). En esa misma línea, Romero (2017) analizó la incidencia de la Planificación Estratégica en la Visión y Misión institucional, y en la calidad de gestión universitaria, es así que, aplicando la metodología cuantitativa, concluye que es indudable la relación existente entre la calidad de gestión y el desarrollo de los planes estratégicos a largo plazo, planes que establecen la visión para alcanzar los propósitos con el trabajo comprometido de cada uno de los actores de la institución (pág. 46)

\section{Plan Integral de Fortalecimiento Académico.}

Es un documento que incluye las estrategias de acción, las mismas pueden variar considerablemente, dependiendo de aspectos o procesos específicos de la gestión que se pretendan mejorar y parte de un diagnóstico para elaborar la planificación, que orienta la 
ejecución de las estrategias, las mismas que serán evaluadas, y de existir debilidades elaborar el plan de mejoramiento continuo, sus dimensiones son: Planificación, Ejecución, Evaluación y Plan de Acción (Completa ,2016, pág. 74). En el mismo sentido, Ladrón de Guevara (2018) expone que evaluación es la recogida de información y el procesamiento de esta para la obtención de resultados, su interpretación orientara la conducción del proyecto, algunos aspectos para la adecuada evaluación son: ser pertinente, planificada, estructurada, ligada a los objetivos institucionales, ser informativa, y contar con respaldo presupuestario (pág.60). Así mismo, se constituyen en un apoyo decisorio para quien gerencie, propiciando actividades de control en cada nivel para comparar lo planificado versus lo ejecutado (Castillo et al., 2019, pág. 71). También Wang (2019) indica que en las universidades la big data, debe protegerse y para esto se constituirá un entorno tecnológico integrador de tres elementos (Universidad, Gobierno, empresa) en un proceso de innovación colaborativa, este procedimiento será evaluado para medir el impacto permanentemente (pág.1).

Del mismo modo para evaluar la calidad del docente, se valoran 5 capacidades fundamentales que son: directiva, interactiva, formativa, motivadora y colaborativa, que son el conjunto de prácticas relacionadas con la dirección, y permiten que se generen sinergias educativas, con la finalidad de tomar decisiones asertivas y trascendentes que conlleven a la mejora continua (García-Garnica, 2018, pág. 141-142). Por lo tanto, el perfil profesional del docente según Arreola (2019) contemplará para su desempeño la aplicación de estrategias de enseñanza actualizadas con el uso de la tecnología para el desarrollo del aprendizaje significativo, que evidencien su nivel de formación y profesionalismo (pág. 6). Se demuestra el profesionalismo y dominio de entornos académicos, y como apoyo, se verá en la necesidad de capacitarse (Orozco Francia, 2017, pág.156). En la misma línea, Stosic (2015) afirma que el docente debe dominar la tecnología educativa para su labor, y esto lo lograra con el manejo de las herramientas de enseñanza al analizar el ritmo de avance, la revisión de contenido mediante resultados inmediatos de evaluaciones para medir el progreso (pág.112). 


\section{Calidad del servicio educativo.}

La calidad es la suma de esfuerzos orientados a alcanzar los estándares como mínimo nacionales, pero lograr esta calidad es un reto y debe ser asumida a través del impulso a la investigación, la mejora de la calidad docente y su real vinculación con la sociedad (Socorro et al., 2017, pág. 53). Alvites (2015) refiere que cada estudiante genera su propia cultura investigativa, y para este objetivo, los semilleros son los espacios universitarios que mejor se ajustan al desarrollo investigativo (pág. 63). Por otro lado, Rappoport y Rodríguez (2019) afirman que los congresos son una oportunidad para intercambiar experiencias, y es que la investigación y la publicación científica son dos actividades íntimamente relacionadas (pág. 7). En esta misma línea, Holmes (2020) promueve la aplicación de un programa de investigación que integra a investigadores por áreas, para analizar profundamente las problemáticas que lleguen a verdaderas alternativas de solución (pág. 517). Todo lo anteriormente expuesto, sobre el proceso educativo se complementa en el ambiente de aprendizaje, en este sentido Quesada-Chaves (2019) afirma que debe ser un espacio con indicadores mínimos de calidad (pág. 6). Los aportes de Harvey y Green (1993, citado en Olaskoaga et al., 2015) que presentan una figura en la que exponen cinco aspectos relacionados directamente con el criterio calidad en la educación superior, en dos aristas, una que identifica los aspectos académicos, desde la perspectiva tradicional y la segunda cobre la calidad educativa y como se evidencia la transformación de los estudiantes desde una perspectiva moderna (pág. 92).

En referencia a la Teoría de la calidad, Terrones (2016) se orienta hacia un cambio en la educación en todas sus modalidades y dimensiones, para superar aspectos negativos, por otros positivos, y precisa satisfacer las expectativas de los estudiantes (párr.15). Por la búsqueda de la calidad educativa, es que se presentan muchos modelos de gestión, en este caso el Modelo de Excelencia y Calidad en la Gestión Empresarial (E.F.Q.M.) que se fundamenta en garantizar la satisfacción de los clientes, por esto se identifican los 3 procesos sustanciales en la organización como son: dirección, ejecución y resultados, relacionados de forma global (Club Excelencia en Gestión, 2017, Párr.2-4). También Torres (2019) indica que el Modelo de Gestión de la calidad total (T.Q.M.) basa sus estrategias en la optimización de todos los procesos, es así como se vuelve una filosofía 
de dirección eficiente, todos son capaces de dirigir sus espacios, todos son responsables de lo que les corresponde (pág.25). Crosby y Bryson (2005, citado en Zavala, 2017, pág. 74) indican que planificar en una institución permite dinamizar las actividades e interrelacionar las funciones entre departamentos, cuya eficiencia garantiza las metas de la institución. Así mismo, la planificación según Argudo et al., (2019) consiste en implementar sistemas administrativos para formular, ejecutar, controlar, realizar seguimientos y evaluar planificaciones institucionales basados en indicadores de control de la gestión para el logro de las metas (pág. 89). En este sentido, una de las características que se destacan sobre la planificación la encontramos en la investigación de Romillo y Polaino (2019) que utilizan un esquema para la gestión en la universidad, y establecen estratégicamente los procesos para el desarrollo de una forma esquematizada en la Pirámide para el desarrollo (PEDI, 2015-2020. pág. 106).

\section{METODOLOGÍA}

\section{Materiales y métodos.}

La investigación fue de tipo aplicada-descriptiva-explicativa, de enfoque mixto, el diseño de investigación que se aplicó es No Experimental de corte transeccional. La muestra seleccionada para la presente investigación es de $\mathrm{N}=249$ que comprendía docentes, estudiantes y personal administrativo, a los que se aplicó la técnica de la encuesta on-line, utilizando (Google-Form), en la investigación se utilizó el cuestionario como un solo instrumento, en el cual se comprendió a las dos variables de estudios con sus dimensiones e indicadores.

\section{RESULTADOS}

Análisis descriptivo

\section{Figura 1}

Los procesos de planificación vigente contribuyen a un adecuado plan de

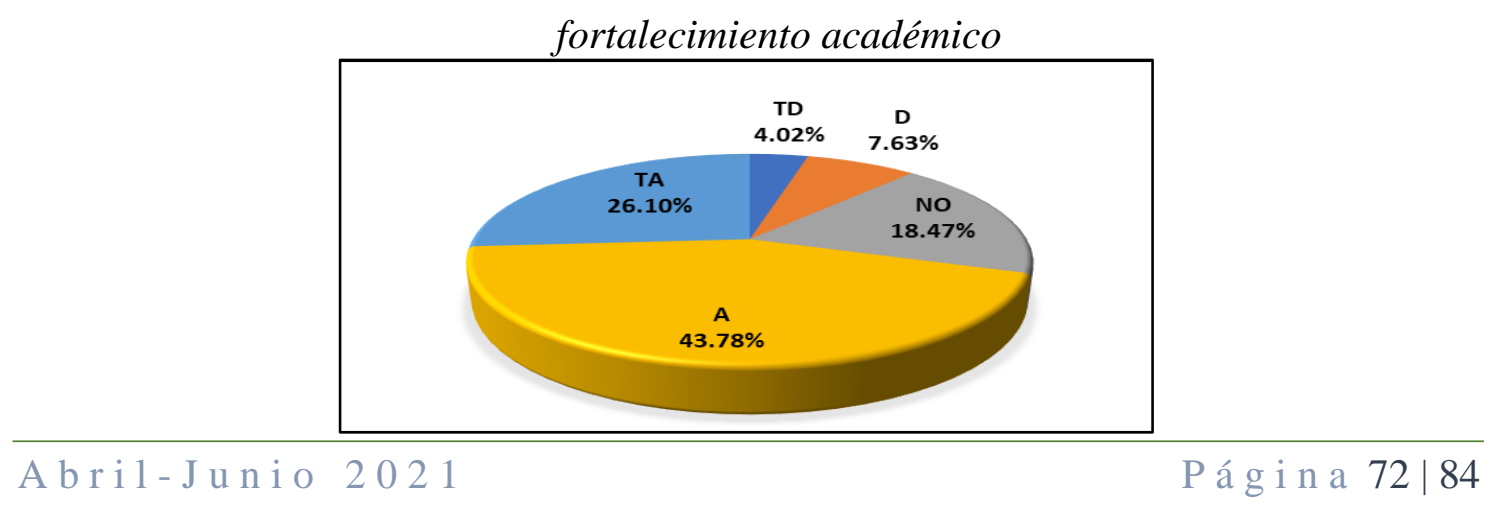


Nota: Con los datos obtenidos, sobre los procesos de planificación vigente contribuyen a un adecuado plan de fortalecimiento académico, un $11.65 \%$ no están de acuerdo con lo afirmado ( $4.02 \%$ TD y un $7.63 \%$ D), asimismo un $18.47 \%$ prefiere No Opinar, mientras que un $69.88 \%$ opinan estar de acuerdo (43.78 \% A y $26.1 \%$ TA).

Fuente: Encuesta de opinión Plan integral de fortalecimiento académico para mejorar la calidad del servicio educativo.

\section{Figura 2}

El actual perfil profesional de los docentes garantiza la calidad del servicio educativo en la institución

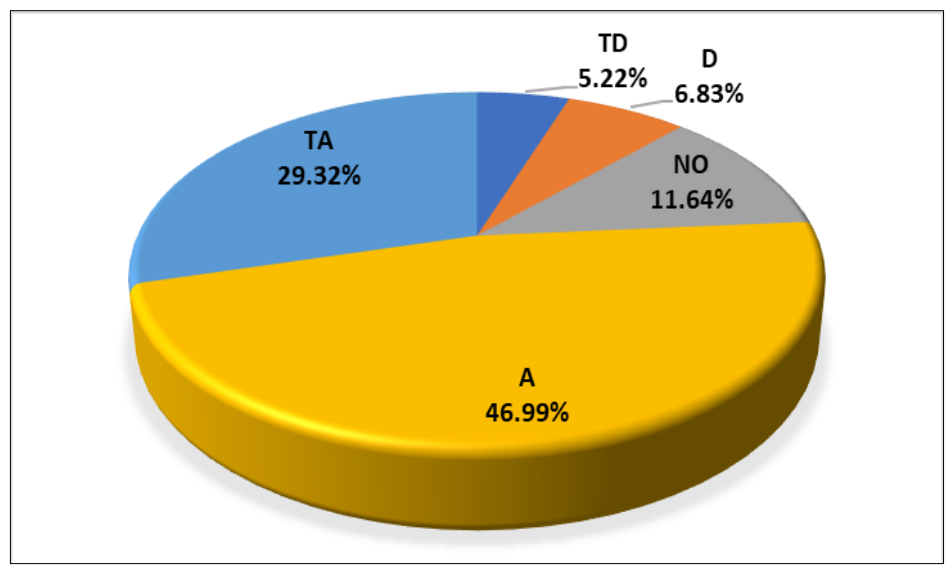

Nota: Respecto a si el actual perfil profesional de los docentes garantiza la calidad del servicio educativo en la institución, el $12.05 \%$ refieren su desacuerdo con lo afirmado (5.22 \% TD y $6.83 \%$ D), asimismo un $11.64 \%$ prefiere No Opinar, mientras que un 77.31 $\%$ expresan su acuerdo con lo expuesto (46.99\% A y $29.32 \%$ TA).

Fuente: Encuesta de opinión Plan integral de fortalecimiento académico para mejorar la calidad del servicio educativo. 


\section{Figura 3}

En las aulas se propicia la investigación formativa como parte de la mejora de la calidad educativa

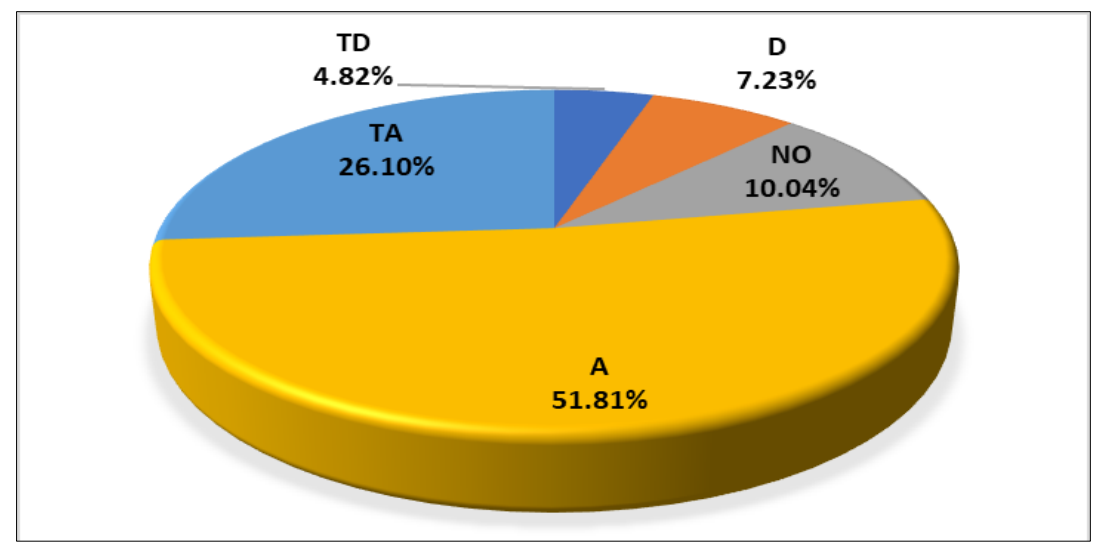

Nota: Sobre si en las aulas se propicia la investigación formativa como parte de la mejora de la calidad educativa, al respecto un $12.05 \%$ manifiestan su desacuerdo con lo expuesto ( $4.82 \%$ TD y $7.23 \%$ D), también, un $10.04 \%$ prefiere No Opinar, mientras que el 77.91 $\%$ muestran su conformidad con lo expuesto $(51.81 \%$ A y $26.10 \%$ A).

Fuente: Encuesta de opinión Plan integral de fortalecimiento académico para mejorar la calidad del servicio educativo.

\section{Figura 4}

La institución viene promoviendo programas de vinculación social con la comunidad

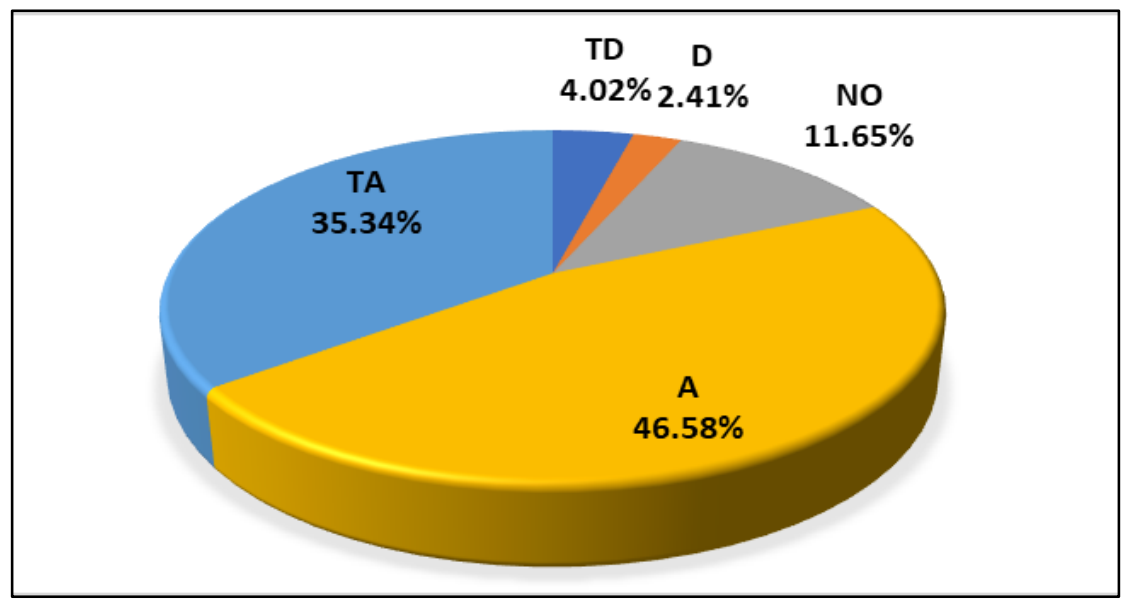

Nota: Respecto sobre si la institución viene promoviendo programas de vinculación social con la comunidad, al respecto un $6.43 \%$ no comparten el enunciado (4.02\% TD y 
$2.41 \%$ D), asimismo un $11.65 \%$ prefiere No Opinar, mientras que el $81.92 \%$ apoya lo afirmado (46.58 \% A y $35.34 \%$ TA).

Fuente: Encuesta de opinión Plan integral de fortalecimiento académico para mejorar la calidad del servicio educativo.

\section{Análisis inferencial: Prueba Chi Cuadrado y Correlación.}

\section{Prueba de Hipótesis General}

$\mathrm{H}_{0}$ : El Plan Integral de Fortalecimiento Académico No se relaciona significativamente con la Calidad del Servicio Educativo en la Universidad Técnica De Babahoyo, Ecuador 2020.

$\mathrm{H}_{1}$ : El Plan Integral de Fortalecimiento Académico se relaciona significativamente con la Calidad del Servicio Educativo en la Universidad Técnica De Babahoyo, Ecuador 2020.

Tabla 1

Pruebas de chi-cuadrado

Valor df

Significación asintótica (bilateral)

\begin{tabular}{llll}
\hline Chi-cuadrado de & 259.4 & 4 &, 000 \\
Pearson & $48^{\mathrm{a}}$ & &, 000 \\
Razón de verosimilitud & 176.0 & 4 &, 000 \\
Asociación lineal por & 30 & 1 & \\
lineal & 140.8 & 1 & \\
\hline N de casos válidos & 82 & & \\
\hline
\end{tabular}

a. 3 casillas $(33,3 \%)$ han esperado un recuento menor que 5. El recuento mínimo esperado es ,53.

\section{Interpretación:}

Como el valor de sig. (valor crítico observado) $0.000<0.05$ rechazamos la hipótesis nula y aceptamos la hipótesis alternativa, es decir que el Plan Integral de Fortalecimiento Académico se relaciona significativamente con la Calidad del Servicio Educativo. 
Análisis de Correlación

Tabla 2

Correlación entre las variables Plan Integral de Fortalecimiento Académico y la Calidad del Servicio Educativo en la Universidad Técnica De Babahoyo, Ecuador 2020.

\begin{tabular}{|c|c|c|c|}
\hline \multicolumn{4}{|c|}{ Correlación de Rho de Spearman } \\
\hline $\begin{array}{c}\mathrm{V} . \\
\text { Independiente }\end{array}$ & $\begin{array}{c}\mathrm{V} . \\
\text { Dependiente }\end{array}$ & $\begin{array}{c}\text { Coeficiente } \\
\text { de } \\
\text { correlación }\end{array}$ &, $867 * *$ \\
\hline $\begin{array}{l}\text { Plan Integral de } \\
\text { Fortalecimiento }\end{array}$ & $\begin{array}{c}\text { Calidad del } \\
\text { Servicio }\end{array}$ & $\begin{array}{c}\text { Sig. } \\
\text { (bilateral) }\end{array}$ & ,000 \\
\hline Académico & Educativo & $\mathrm{N}$ & 249 \\
\hline
\end{tabular}

\section{Interpretación:}

En la tabla 2 se evidencia que la variable Plan Integral de Fortalecimiento Académico tiene una relación positiva alta con la variable Calidad del Servicio Educativo $($ rho $=.867 * *)$, lo cual permite rechazar la hipótesis nula que postula la inexistencia de una relación entre las variables.

\section{DISCUSIÓN DE RESULTADOS}

De los resultados presentados en la figura 1, sobre si los procesos de planificación vigente contribuyen a un adecuado plan de fortalecimiento académico, un $43.78 \%$ están de acuerdo, lo que está en correspondencia con lo manifestado por Completa (2016), quien establece que un plan, es la hoja de ruta que debe seguir una institución para mejorar sus procesos basados en el análisis y toma de decisiones (pág. 4). En la figura 2, sobre si el actual perfil profesional de los docentes garantiza la calidad del servicio educativo en la institución, al respecto un 46.99 \% refieren estar de Acuerdo y el 29.32\% están Totalmente de Acuerdo con lo manifestado. Estos resultados se relacionan con lo manifestado por García-Garnica (2018) quien afirma que la calidad del docente se 
evidencia en dos aspectos del profesional, las acciones evidenciables en su desempeño y el cúmulo de conocimientos certificados por instituciones de formación y de experiencias acumuladas en su trayectoria (pág. 141).

De acuerdo con los datos desagregados en la figura 3, sobre si en las aulas se propicia la investigación formativa como parte de la mejora de la calidad educativa, al respecto un $51.81 \%$ refieren estar de Acuerdo y $26.1 \%$ están Totalmente de Acuerdo con lo manifestado. Esto está en correspondencia con lo que Socorro et al. (2017) afirman en relación con la Investigación Científica en el contexto universitario que tiene como fin desarrollar conocimientos, impulsar avances con carácter innovador que permitan solucionar problemas del contexto (pág. 53). Así mismo, Rappoport y Rodríguez (2019) afirman que los congresos son una oportunidad para aprender de los ponentes, y es que la investigación y la publicación científica son dos actividades íntimamente relacionadas, puesto que la primera no culmina cuando se obtienen los resultados o se entrega y defiende un informe final; sino que llega a su fin cuando se socializa el nuevo conocimiento. Por su parte, los Indicadores de Impacto, consideran la cantidad de citaciones que obtienen, y a partir del reconocimiento otorgado por otros investigadores (pág. 7).

En la figura 4 se expresan las opiniones sobre si la institución viene promoviendo programas de vinculación social, y al respecto un $46.58 \%$ están de acuerdo y el $35.34 \%$ están totalmente de acuerdo y se relacionan con lo manifestado por Cisneros y Mendoza (2018) que opinan que los proyectos de vinculación demostrarán compromiso mediante las funciones sustantivas propias de la actividad universitaria (Pág. 55).

\section{PROPUESTA}

\section{Titulo}

\section{Plan integral de fortalecimiento académico para la mejora continua de la Universidad}

\section{Técnica de Babahoyo.}

Un plan de fortalecimiento académico, tiene muchas formas de presentación, pero en la presente propuesta consta de tres fases que son: planificación de los procesos, ejecución de las estrategias y evaluación de indicadores de resultados, con esta estructura, se han establecido los elementos fundamentales que deberán fortalecerse, y que se encuentran 
fuertemente ligados entre sí, se evidencia la necesidad de establecer un esquema integrado que debe responder a la formación integral que necesitan los estudiantes en cada área del conocimiento, por ello, para alcanzar la calidad del servicio educativo, como propuesta se presenta el Plan de Fortalecimiento Académico para la Mejora Continua, tal como se aprecia en la figura 5

\section{Figura 5}

Estructura del Plan de Fortalecimiento Académico para la mejora continua de la U.T.B

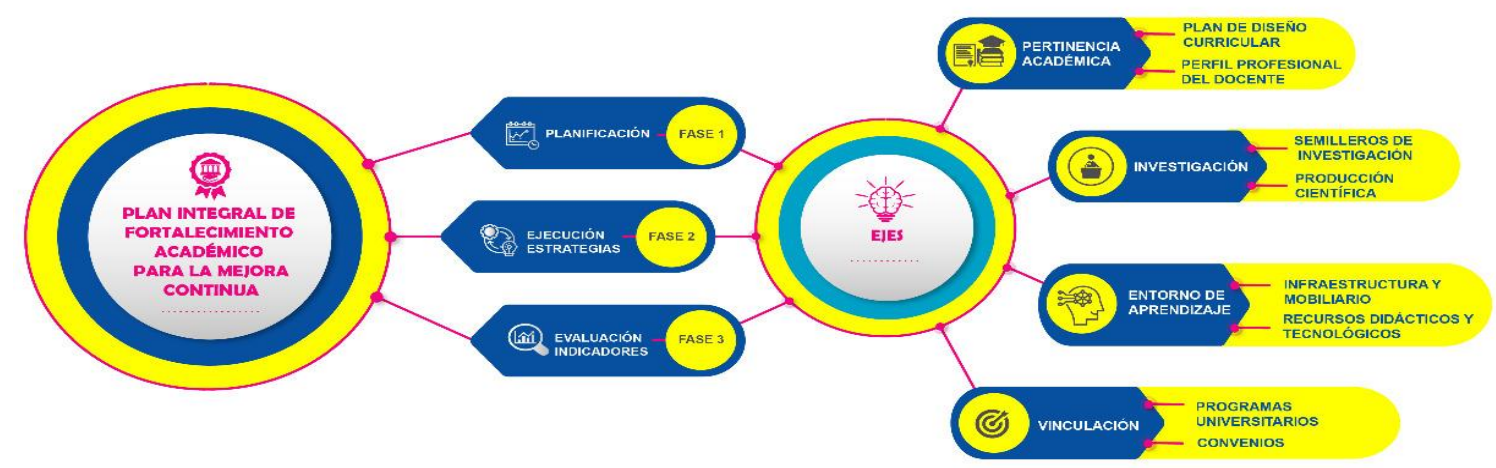

El objetivo general de la propuesta es: Proponer un plan de fortalecimiento académico en base a la mejora continua para garantizar la calidad del servicio educativo, y para alcanzarlo, se deberá Elaborar la planificación para asegurar el fortalecimiento académico, Ejecutar las etapas del Plan de fortalecimiento Académico y evaluar el impacto de eficacia y eficiencia de la aplicación del plan de fortalecimiento Académico.

\section{CONCLUSIONES}

Se elaboró el plan integral de fortalecimiento académico, cuya estructura interna permite viabilizar el accionar del plan en mención, para promover la mejora de la calidad del servicio educativo que brinda la Universidad Técnica de Babahoyo.

Existe la necesidad, que los docentes cuenten con formación académica, experiencia profesional y capacitaciones acorde a las asignaturas asignadas, tal como opina el 86,31\% de los encuestados (tabla 4); así mismo, respecto a los procesos de investigación formativa y producción científica de los docentes, se evidencia que los docentes deben potenciar la cultura investigativa; además frente a los entornos de aprendizaje con los que actualmente cuenta la institución, queda claro que estos merecen mayor nivel de atención, actualización de los equipos, materiales y recursos didácticos, así como 
también, adecuar los espacios universitarios, y en lo relacionado a los programas de vinculación social, los encuestados se pronunciaron a favor un $81.92 \%$ indicando que la universidad debe acercarse a la sociedad, a través de convenios interinstitucionales que permitan el desarrollo de los proyectos de investigación acorde a los requerimientos sociales.

Se identificaron los factores que están influyendo en la calidad del servicio educativo, los que de cierto modo no están alejados a las funciones propias de la actividad universitaria, sin embargo, estas actividades deben completar el ciclo administrativo que inicia con una pertinente planificación, y que de acuerdo con las opiniones de un 69.88\% (tabla 3) se deberán fortalecer.

Para el diseño del plan integral de fortalecimiento académico se contempló una estructura de 3 fases que agrupan los procesos más relevantes y a la vez, complementarios entre sí, como son la planificación pertinente, la ejecución de estrategias educativas y la evaluación de los procesos, que garantizaran el mejoramiento continuo, con lo que se potenciara la calidad del servicio educativo de la Universidad Técnica de Babahoyo.

\section{REFERENCIAS BIBLIOGRÁFICAS}

Alvites, C. (2015). Creación e implementación de semilleros de investigación en la Dirección Universitaria de Educación a Distancia de la Universidad Alas Peruanas. Revista semestral de divulgación científica Hamut'ay. https://dialnet.unirioja.es/servlet/articulo?codigo $=5665696$

Araujo, M. (2016). Pertinencia académica profesional en la formación de docentes para Educación Inicial en el estado Trujillo: Una propuesta a partir de la investigación-acción.

Revista

Educere.

20

https://www.redalyc.org/jatsRepo/356/35646429009/html/index.html

Arreola, R. (2019). Formación y evaluación docente basada en un perfil por competencias. Una propuesta desde la práctica reflexiva. Revista Educación, 43 (2). $\begin{array}{llll}\text { Universidad de Costa } & \text { Rica. }\end{array}$ https://revistas.ucr.ac.cr/index.php/educacion/article/view/30898

Barreno, M., Barreno, Z. y Olmedo, A. (2018). La educación superior y su vinculación con la sociedad: referentes esenciales para un cambio. Revista Universidad y Sociedad. pp.40-45. http://scielo.sld.cu/pdf/rus/v10n3/2218-3620-rus-10-03-40.pdf 
Becerra, A. (2019). Las cinco cuentas pendientes con la universidad española. Cadena

Ser. https://cadenaser.com/ser/2019/09/22/sociedad/1569137230_616613.html

Blog Calidad y Excelencia (27 de noviembre de 2019). EFQM 2020 Principales cambios en el nuevo Modelo Europeo de Excelencia Madrid. ISOTools Excellence. https://www.isotools.org/2019/11/27/efqm-2020-principales-cambios-en-el-nuevomodelo-europeo-de-excelencia-se-renueva/

Bournissen, J. (2019). Modelo pedagógico para la Facultad de Estudios Virtuales de la Universidad Adventista de La Plata. Argentina. https://www.tesisenred.net/bitstream/handle/10803/402708/tjmb1de\%206.pdf?sequence $\equiv 1 \&$ isAllowed $=\mathrm{y}$

Castañeda, G. (2015). Percepción de la planificación de unidades didácticas de docentes del área de Ciencia, Tecnología y Ambiente secundaria en instituciones educativas de Abancay, Apurímac. Universidad Peruana Cayetano Heredia. Perú. http://repositorio.upch.edu.pe/handle/upch/125

Castillo, J., Erazo, J., Narváez, C. y Torres, M. (2019). Auditoría de gestión y su incidencia en la eficiencia y eficacia de las operaciones de una empresa comercial. Visionario Digital, 3(2.1.), 159-188. https://cienciadigital.org/revistacienciadigital2/index.php/VisionarioDigital/article/view/ 551? articlesBySameAuthorPage $=3$

Cisneros, P. y Mendoza, K. (2018) Vinculación Universidad - Sociedad: espacio para generar creatividad e innovación. Revista Killkana Sociales. 2(2), pp. 53-58. https://doi.org/10.26871/killkana_social.v2i2.304

Club Excelencia en Gestión (2017). Transformarse no es una opción. Es una necesidad en todos los sectores $y$ en todos los tamaños de empresas. https://www.clubexcelencia.org/conocimiento/modelo-efqm

Completa, E. (2016). State capacity, capacity gaps and institutional $\begin{array}{llll}\text { strengthening. } & \text { Análisis } & \text { Político, 29(87), } & \text { 60- }\end{array}$ http://www.scielo.org.co/pdf/anpol/v29n87/v29n87a03.pdf

Consejo de Evaluación, acreditación, y Aseguramiento de la Calidad de la Educación Superior [CEAACES]. (diciembre de 2017). Modelo Genérico de Evaluación del Entorno de Aprendizaje de Carreras. 
https://www.utmachala.edu.ec/archivos/filesportal/2019/EVALUACION/Modelo_Gene $\underline{\text { rico_Evaluacion_entorno_aprendizaje_dic_2017.pdf }}$

Espinoza, C. (2016). Calidad de la educación e índices de gestión en relación con el presupuesto de las universidades del Ecuador en el año 2015. Revista Universidad y Sociedad. pp. 210-217. http://scielo.sld.cu/pdf/rus/v8n2/rus27216.pdf

Galván, F. (12 de abril de 2019). El problema de la educación superior en México es de calidad no de cantidad. Portal electrónico merca20.com. https://www.merca20.com/elproblema-de-la-educacion-superior-en-mexico-es-de-calidad-no-de-cantidad/

García, D. (2019). La formación de profesionales socialmente responsables en la universidad: una utopía posible en el currículo. Universidad de los Andes. https://bv.unir.net:3555/es/lc/unir/titulos/118374

García-Garnica, M. (2018). Prácticas eficaces de liderazgo dirigidas a apoyar la calidad docente en los CEIP públicos de Andalucía. La percepción de directivos y maestros. REICE. Revista Iberoamericana sobre Calidad, Eficacia y Cambio en Educación. https://revistas.uam.es/reice/article/view/9714

Holmes, B. (2020). Re-imagining Research: A Bold Call, but Bold Enough? Comment on "Experience of Health Leadership in Partnering with University-Based Researchers in Canada: A Call to 'Re-Imagine' Research'. International Journal of Health Policy and Management, 9 (12), 517-519. DOI: https://doi.org/10.15171/IJHPM.2019.139

Ivanova, D., Goray, O., Horbachova, N., Krukovska, I. y Poplavska, S. (2020). The objectives and practical aspects of quality assurance system of higher education. International Journal of Higher Education, 9(7), 119-129. https://doi.org/10.5430/ijhe.v9n7p119

Ladrón de Guevara, M. (2018). Programación, ejecución y difusión de proyectos educativos en el tiempo libre. UF1948. Editorial Tutor Formación. Madrid. https://bv.unir.net:3555/es/ereader/unir/105976

Miyahira, A. (2019). La investigación formativa y la formación para la investigación en el pregrado. Revista Médica Herediana, https://revistas.upch.edu.pe/index.php/RMH/article/view/1010?articlesBySameAuthorP $\underline{\text { age }=2}$ 
Modelo de evaluación externas de universidades y escuelas politécnicas. (2019). CASES.

Consejo de aseguramiento de la calidad de la educación superior. https://www.caces.gob.ec/wp-content/uploads/downloads/2019/12/3.-

Modelo_Eval_UEP_2019_compressed.pdf

Montero, O. (15 de enero de 2018). Reforma urgente a nuestro sistema de educación superior. Diario El telégrafo.

https://www.eltelegrafo.com.ec/noticias/columnistas/15/reforma-urgente-a-laeducacion-superior

Olaskoaga, L., Marúm, E., y Partida, I. (2015). La diversidad semántica y el carácter político de las nociones de calidad en la Educación Superior de México. Revista de la

$\begin{array}{llllll}\text { Educación } & \text { Superior. } & \text { Vol. } & \text { XLIV } & \text { (1); } & \text { No. }\end{array}$ http://www.scielo.org.mx/pdf/resu/v44n173/v44n173a4.pdf

Orozco, A. (2017). El impacto de la capacitación. México, D.F, México: Editorial Digital UNID.https://descargalibrosgratis.club/el-impacto-de-la-capacitacion/

Quesada, M. (2019). Condiciones de la infraestructura educativa en la región pacífica central: los espacios escolares que promueven el aprendizaje en las aulas. Revista Educación, 43 (1), 1-35. https://www.scielo.sa.cr/pdf/edu/v43n1/2215-2644-edu-43-0100293.pdf

Rappoport, S. y Rodríguez, M. (2019). Presentación de Calidad de la Educación: Debates, investigaciones y prácticas. Editor: Dykinson. UAM. Departamento de Didáctica y Teoría de la Educación. https://www.dykinson.com/libros/calidad-de-la-educaciondebates-investigaciones-y-practicas/9788413241821/

Rodríguez, M., Rivadulla, J. (2017). Percepciones sobre la educación de personas adultas. Análisis de la formación, la práctica docente y el perfil profesional. Revista Educar, 54/2 431-448. https://doi.org/10.5565/rev/educar.898

Romero, P. (2017). La Planificación Estratégica y su influencia en la Calidad de la Gestión Educativa de la Universidad Técnica de Babahoyo. Universidad Nacional Mayor de San Marcos. Perú. https://revistas.utb.edu.ec/index.php/sr/article/view/56 Romillo, A. y Polaino, C. (2019). Aplicación del modelo de gestión pirámide del desarrollo universitario en la universidad de Otavalo, Ecuador. 
https://scielo.conicyt.cl/scielo.php?script=sci_abstract\&pid=S0718-

$\underline{50062019000100003 \& \operatorname{lng}=\mathrm{es} \& \mathrm{nrm}=\mathrm{iso}}$

Rubio, M. Vilá, R. y Berlanga, S. (2015). La investigación formativa como metodología de aprendizaje en la mejora de competencias transversales, Procedia - Social and $\begin{array}{llllll}\text { Behavioral } & \text { Sciences } & 196 . & \text { pp.177 } & - & 182\end{array}$ https://www.sciencedirect.com/science/article/pii/S1877042815040239

Sanz, E. (s.f.). Las fases de la ejecución de un proyecto. Consultores Documentales.https://sorprendemos.com/consultoresdocumentales/?p=347

Salas-Rueda, R. (2018). Use of the Deming cycle to ensure quality in the educational process on mathematics. CIENCIA UNEMI, 11(27), http://ojs.unemi.edu.ec/index.php/cienciaunemi/article/view/781

Socorro, A., Espinosa, C. y León, J. (2017). Uso de la información científica y tecnológica en la investigación y la innovación. Cienfuegos, Cuba: Editorial Universo Sur. https://bv.unir.net:3555/es/ereader/unir/71782

Stosic, L. (2015), College of professional's studies educatord, Aleksinac Serbia. The importance of educational technology in teaching. Vol.3. https://dialnet.unirioja.es/servlet/articulo?codigo=5109037

Terrones, E. (24 de abril de 2016). La teoría de la calidad educativa y las universidades. Blog de Eudoro Terrones Negrete. http://eudoroterrones.blogspot.com/2016/04/la-teoriade-la-calidad-educativa-y-las.html

Torres, A. (11 de junio de 2018). El éxito del Modelo Educativo en Canadá, donde los profesores no se eligen en oposiciones. El Periódico El País. https://elpais.com/economia/2018/05/28/actualidad/1527526183_441482.html

Torres, E. (20 de octubre de 2019). Impacto de total quality management en el desempeño organizativo. Revista Investigación \& Desarrollo. http://www.upb.edu/revistainvestigacion- desarrollo/index.php/id/article/view/197

Torres, S., Ruíz, D. y Meza, L. (21 de febrero de 2017). Infraestructura y equipamiento tecnológico en los doctorados consolidados de Conacyt: una mirada desde las políticas públicas y los académicos. Revista iberoamericana de educación superior. vol.8. México. https://www.redalyc.org/jatsRepo/2991/299149615001/index.html 
Universidad Técnica de Babahoyo (6 de mayo de 2016). Acta de la sesión extraordinaria

$\begin{array}{lllll}N^{\circ} & 07 & \text { del } & \text { H. } & \text { Consejo Universitario. }\end{array}$

https://drive.google.com/file/d/OBwIXXoT3SKaSeGQ0NWt2djFGOUk/view

Wang, L. (2019). Analysis of application of big data in college education management.

Paper presented at the Journal of Physics: Conference Series, 1314(1). doi: https://doi.org/10.1088/1742-6596/1314/1/012220

Zhang, Q., Jiang, S., Liu, R., \& Liu, H. (2020). An Integrated Decision-Making Model

for Analyzing Key Performance Indicators in University Performance Management.

Matemáticas 2020, 8, 1729. http://dx.doi.org/10.3390/math8101729

\section{PARA CITAR EL ARTÍCULO INDEXADO.}

Intriago, G. C., Mendiburu Rojas, A. F., \& Oviedo Rodríguez , M. D. (2021). Plan integral de fortalecimiento académico para mejorar la calidad del servicio Educativo. Magazine De Las Ciencias: Revista De Investigación E Innovación, 6(2), 64-84. https://doi.org/10.33262/rmc.v6i2.1252 